\title{
Exploiting Tag Clouds for Database Browsing and Querying
}

\author{
Stefania Leone, Matthias Geel, Corinne Müller, and Moira C. Norrie \\ Institute for Information Systems, ETH Zurich \\ CH-8092 Zurich, Switzerland \\ \{leone,geel, norrie\}@inf .ethz.ch
}

\begin{abstract}
Querying and browsing of databases is a task exclusively done by experts that have mastered the query language and are familiar with a database's schema. We show how tag clouds can be used alongside more traditional query languages and data visualisation techniques as a means for browsing and querying databases by both experts and non-expert users. Our approach is based on a general, extensible framework that supports different modes of visualisation as well as different database systems. We have validated our prototype with a user study that has shown how non-experts were able to browse and retrieve data that usually would only be possible by means of queries.
\end{abstract}

Keywords: tag cloud, data visualisation, database interface.

\section{Introduction}

Tag clouds are becoming extremely popular as a means of providing visual summaries of collections of documents. Although very simple, they can be used to support search, browsing and recognition as well as forming and presenting impressions [1]. The presentation and layout of tags can be controlled so that features such as the size, font and colour can be used to give some measure of the importance of a given tag, while the positioning of tags may be based on pure aesthetics, alphabetical sorting or some form of relationship between tags.

While tag clouds have been widely used in Web 2.0 applications for visualising user-generated tags and folksonomies of web sites such as Flickn, more recently they have been introduced into various application domains as a means of summarising and visualising specific domain information. For example, in [2] tag clouds were used as a visual augmentation of employee profile pages, while [3] use them to summarise research activities.

Given the flexibility of tag clouds in terms of information representation together with the simplicity of the associated style of navigation, it is natural that database researchers should consider exploiting the concept of tag clouds to address the longstanding problems of database usability 4. The use of a query language requires the user to master not only the query language but also the

\footnotetext{
${ }^{1}$ http://wWw.flickr.com
} 
database schema. To allow users to view the data in a natural way, a higher-level presentation of the database content such as a visual schema browser and query interface is needed.

Our goal was to investigate the extent to which tag clouds could be exploited to support database browsing and querying, either by replacing existing query languages and other modes of data visualisation or being used alongside them. Our tag clouds therefore mainly represent data and metadata values rather than terms occurring within the data. To support our investigations, we have developed a general, extensible framework that supports different modes of data visualisation, including customisable tag clouds. We have also designed it so that different types of databases can be accessed, and currently have implementations for both object databases and relational databases.

A key advantage of the tag cloud approach is that it is data-driven rather than schema-driven which is particularly beneficial to users with no experience of databases and query languages. Our initial user studies have shown that even users with low computer literacy and no previous experience of tag clouds were able to find the results of non-trivial queries using our system. At the same time, expert users also gave favourable feedback about the system.

In Sect. 2, we discuss the background to this work before going on to present the details of our approach in Sect. 3. We then provide an overview of the system that we have implemented in Sect. 4. In Sect. 5, we present the user study and its results. Open issues and directions of future research are discussed in Sect. 6 before giving concluding remarks in Sect. 7 .

\section{Background}

While database technology has made great advances in the past decades, database usability has not improved a lot and database systems are still known for being hard to access and query [4. Query languages such as SQL and XQuery are the current means provided by database systems to allow users to access and retrieve data. While these languages are powerful, they require users to not only master the query language but also to fully comprehend the schema of the database. Thus, at present, the capabilities of database systems can only be fully exploited by expert users. End users usually access only structured data by means of special purpose applications built on top of databases, where the data access is constrained by the specific user interface and the functionality provided. Consequently, few general computer users take the step of actually using database software directly to help them create and access personal information.

It has therefore been a longstanding challenge to develop interfaces that can allow non-expert users to access a database without having detailed knowledge about the underlying technology or schema design. To allow users to view the data in a natural way, a higher-level presentation of the database content, such as a visual query interface, is required. A lot of research has already been done in the area of visual query languages [5], but so far such research has tended to have an impact only in specific application domains. In more recent work 4], Jagadish 
et al. presented a system that offers a set of user interfaces for an XML database including a traditional XQuery interface, a keyword search, a natural language interface, a form-based interface and a visual query editor. Since they found that users had difficulties locating elements of interest in the schema tree, they experimented with schema summarisation as well as schema-free approaches.

In contrast, one of the effects of Web 2.0 is that visualisation paradigms such as tag clouds and faceted browsing are now in widespread use for searching large data collections of user-generated content. These interfaces are targeted to support end users in the tasks of browsing and searching data collections in an easy and intuitive way, and are used by non-experts and experts alike. In some cases, a more advanced interface may be provided to support more complex searches, but the simpler visual interfaces will suffice for the majority of queries.

While tag clouds originally emerged for browsing user-generated folksonomies, they have become popular for visualising data of various types. For example, in [2], they describe a social tagging extension to an employee directory application, where employees can tag each other and employee profiles were extended with tag clouds. Each employee profile got enriched with two tag clouds, an incoming and outgoing tag cloud, where the incoming tag cloud visualised the tags assigned to that person from their co-workers and the outgoing tag cloud summarised the terms used by that person to tag their co-workers. Their user studies showed that people tag other people as a form of contact management, to see all the people associated with a project, or to locate experts in a particular area. They also found that the tags were considered by users as giving accurate descriptions of their interests and expertise. Similarly, in [3], we experimented with tag clouds that provide visual summaries of researchers' activities to promote awareness within research groups and institutes. Each researcher was associated with a tag cloud generated automatically based on the documents that they read and wrote. These tag clouds were integrated into an ambient information system deployed within a research group. A user study showed that researchers could recognise other members of their research group based on their tag cloud. At the level of an institute, participants of the study were able to recognise a research group based on an individual's tag cloud but not that individual. This meant that, through a common visualisation scheme, awareness of the activities of individual researchers and research groups could be promoted at a level meaningful to different users.

Tag clouds have also been proposed as a means of summarising and refining the results of keyword searches over structured data as presented in 667. In this case, the term data cloud is used to refer to their particular adaptation of tag clouds for summarising keyword search results. Data clouds were implemented as part of CourseRank, a social tool to access official university information and statistics, such as course descriptions, grade distributions and course evaluations, as well as user-generated information, such as course ratings, comments, questions and answers. Students could use the tool to search for classes, give comments and ratings, and also organise their classes into a personalised schedule. An interesting feature of their approach is that since it was developed for 
relational databases, the developer of a data cloud application specifies how application entities can be composed from the relations in the database in order that keyword search can be applied to entities rather than simple attributes or tuples. The keyword search is based on a traditional information retrieval approach where entities are considered as documents and attribute values as weighted terms.

Another project that uses tag clouds for summarising query results is PubCloud [8] for searching the PubMed biomedical literature database. In this case, the tag clouds are generated from words extracted from the abstracts returned by the query. In 9], an approach using multiple synchronised tag clouds has been proposed to summarise, browse and compare search results over clinical trial data. This approach is similar to the one of faceted browsing in that browsing and querying are combined and results are refined.

In [6], data clouds for specific application domains rely on entity construction of the underlying data done by the cloud application developer, while [9] is only targeted to the domain of clinical trial data and their specific categorisation scheme. In contrast to these approaches which focus on domain-specific information visualisation of data sources, our aim is to strive for a more general approach, where tag clouds can be exploited to browse data in a generic way by offering synchronised tag clouds for browsing both schema and data.

\section{Data Browser}

Our general goal was to provide both non-expert and expert users with a userfriendly and intuitive interface to traditional databases by exploiting the concept of tag clouds and adapting them to the process of querying and browsing structured data. In the Web, the tags within a tag cloud are usually hyperlinks that lead to the collection of items that are associated with the tag. Tag clouds are graphically appealing due to different visualisation features. Tag cloud features include text features, such as the tag content, the size, font style and colour as well as the positioning and order of tags in a cloud. A lot of studies, such as 10 111 have experimented with tag cloud features and positioning and their impact on users. According to [101], font size, font weight and intensity are the most important features. While topic-based layouts of tags can improve search performance for specific search tasks compared to random arrangements, they still perform worse than alphabetic layouts according to [1].

We adapted these concepts to browse structured data where tags represent attribute values. Clicking on a tag initiates a selection for data items with the corresponding attribute value. In the case of object databases, the result would be a collection of objects, while in the case of a relational database it would be a collection of tuples, i.e. a relation. It is possible to form tag clouds from combined attribute values or to combine the display of various attributes by using the tag content to represent one attribute and the colour of a tag to indicate the value of another attribute. Using visual features such as the colour of a tag to represent a specific value of an attribute is appropriate only when the number of distinct values is small. 
We use these concepts to browse both data and metadata and have even experimented with a mix of metadata and data within tag clouds as well as with synchronised browsing. We define a data source to be a set of data collections, where each collection contains data items of a specific type. These collections are either class extents or sets of objects of a specific type in object databases, while they are relations in relational databases.

We now explain these concepts further by means of an example based on a database with information about contacts and their locations. Figure 1 gives an overview of the data model using ER notation. Contacts can either be persons or organisations and are located at specific locations. Persons are further categorised into private contacts, ETH persons and persons that previously worked at ETH. Persons work for organisations and organisations can be part of larger organisations. Note that in an object database representation of this model, the metadata for the entities will map into two concepts, namely the object types and the collections of objects. Thus, contacts would be represented by a type Contact and a collection Contacts.

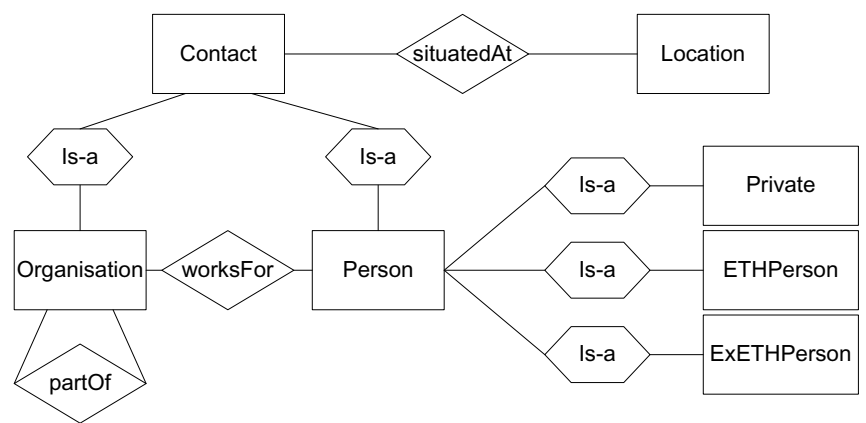

Fig. 1. Contacts Model

We have experimented with different visualisations of both data and metadata. In an earlier version, data was summarised using a tag cloud and metadata collections could be selected by means of drop down menus. In a more recent version, the metadata itself has been visualised by a tag cloud as shown with the screenshot in Figure 2, On the left hand side of the screenshot, the schema tag cloud summarises the names of the various collections of data items within the database. The default is to have the size of the tags represent the relative cardinality of the collection. To distinguish the tag clouds that represent data and metadata, we will refer to these as data clouds and schema clouds, respectivley.

As will be explained later, we implemented several different data adapters on top of relational and object-oriented database systems. A user can start browsing a database either by entering a database-dependent query expression in the window below the tag clouds or by selecting one or more of the tags in the schema cloud. When clicking on a collection tag, the data cloud on the right hand side of the figure is generated. In the example shown in Figure 2, the user has selected the Contacts tag in the schema cloud, which triggered the generation 


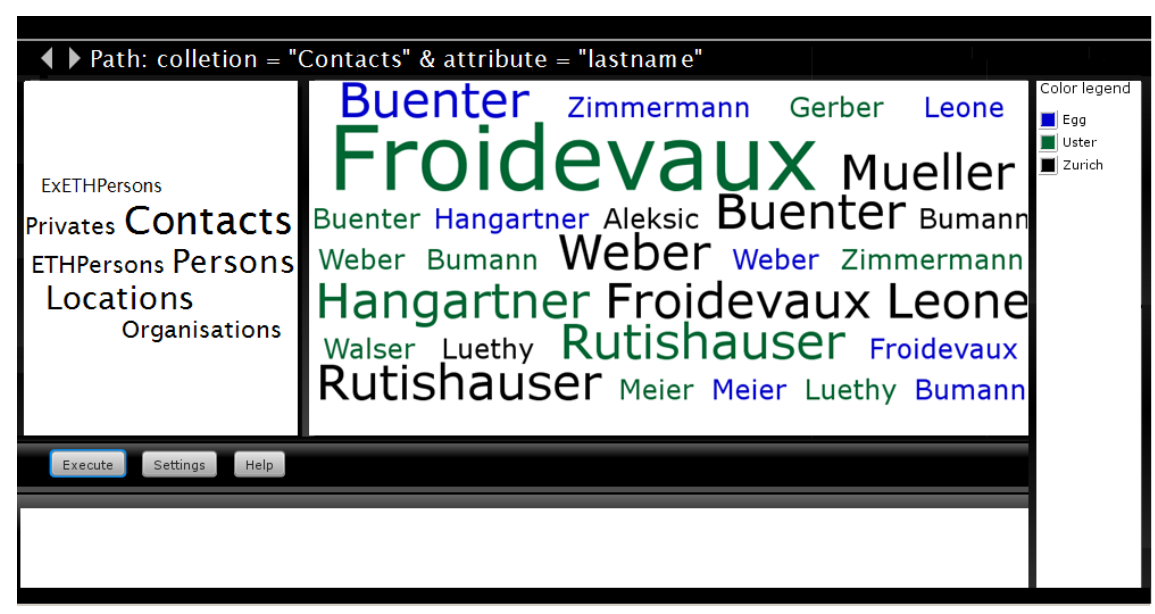

Fig. 2. Schema and Data Browsing

of the data cloud where contacts are summarised according to their last name. This is also indicated by the navigation path shown on top of the screenshot. In addition, contacts can be further grouped by a second attribute, in this case the city attribute which is visually represented by different colours. The lighter tag Froidvaux represents the number of objects with last name Froidvaux that live in Uster, while the black tag Froidvaux represents the objects with last name Froidvaux that live in Zurich. As one can see from the colour legend on the right hand side of the figure, each distinct attribute value of the city attribute is assigned a specific colour. By default, the first non-unique string attribute of each collection is used for its visualisation as a data cloud. However, the user can also specify this by means of a simple selection of attributes through check boxes. Alternatively, one can display the attributes themselves as a schema cloud and allow the users to select one or more attributes as tags. In this way, we support synchronised browsing across the metadata and data through the adjacent tag clouds.

The size of a tag in the data cloud represents how many data items have that particular attribute value. In this way, a data cloud can be considered as a visualisation of the attribute value frequency. The user can now click on a tag and further refine their selection. When hovering over a lastname tag, a user gets detailed information about the number of objects that have this attribute value, or in the case of only a single object, we get the set of attribute values.

A second example of such a user interaction involving the browsing of the schema and the process of refining a query is illustrated in Figure 3. In a first step, the user selects the collection to be queried from a menu. Assume in this case it is the Contacts collection. Schema browsing allows the user to then select and combine attributes not only from the currently selected data items, but also from those associated with them. Assume that the user is interested in the Locations of contacts, particularly the city names where they live. Then from 
Step 1: Browse Schema

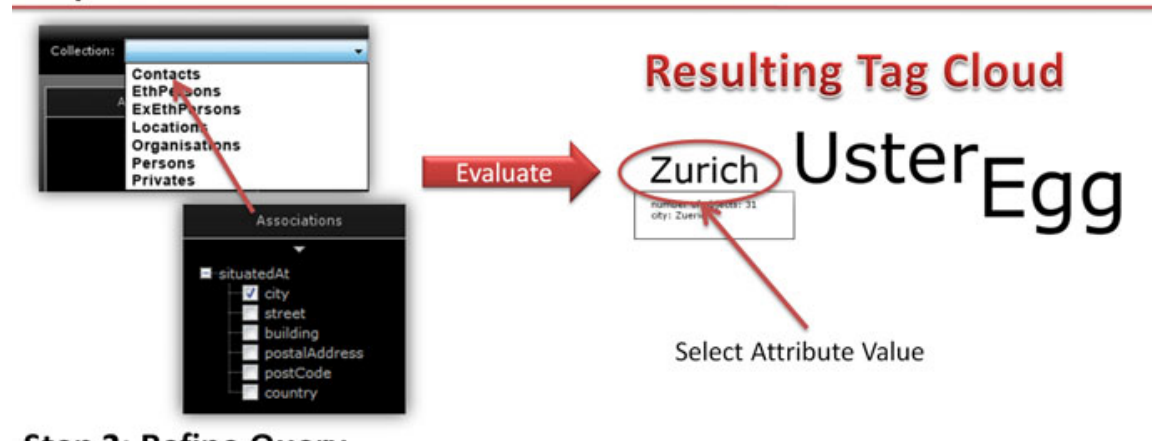

Step 2: Refine Query

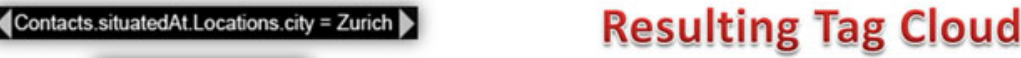
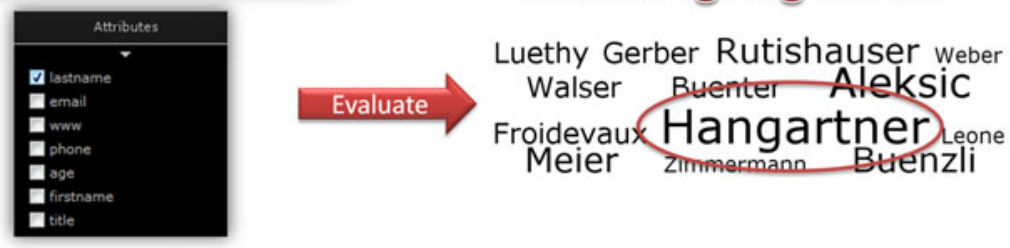

Fig. 3. Interactive Query Formulation

a further menu of associations over Contacts, they could select the SituatedAt association that relates contacts to locations. The user is then provided with a list of the attributes of location objects for them to select. The figure shows the data cloud resulting from a selection of the city attribute. The result is displayed as a data cloud whose terms are weighted according to the occurrences of contacts living in the same city. The user now has several possibilities for how to proceed. They could either start exploring other parts of the schema, display a different selection of attributes of the same entities or they can further refine the current query. In the second step, the example illustrates the latter possibility where the query has been constrained to contacts living in Zurich by selecting the corresponding tag within the result of step 1. In this case, the lastname attribute of Contacts objects is selected to be displayed in the data cloud. From the resulting data cloud, it is easy for the user to gain an overview of the contacts living in Zurich and to see that Hangartner has the most occurrences.

These two user interaction examples illustrate the kind of queries that can be posed by means of tag cloud browsing. The selection of attributes by the user prior to the creation of the tag cloud corresponds to a concatenation of attribute values before the resulting list is grouped by frequency. A click on one of those tags immediately leads to an extension of the query with a predicate ensuring the currently displayed attribute is fixed to that particular tag value for all subsequent queries. This cycle of attribute selection and predicate generation 
can be arbitrarily repeated by the user. However, due to the nature of tag clouds, attributes with a large value range or unique attributes are not very well suited for selection. Furthermore, join operations are supported by explicit traversal of associations when the user navigates between collections. Because of that direct way of interacting with real data, it is not possible to create range queries or any other open queries. For those kinds of queries, we offer a query field. In terms of SQL, the queries that can be answered by our system can be summarised as a selection of concatenated attributes retrieved by (possibly) nested joins with WHERE clauses that contain AND-chained equality predicates. The results are then grouped by frequency and displayed as a tag cloud.

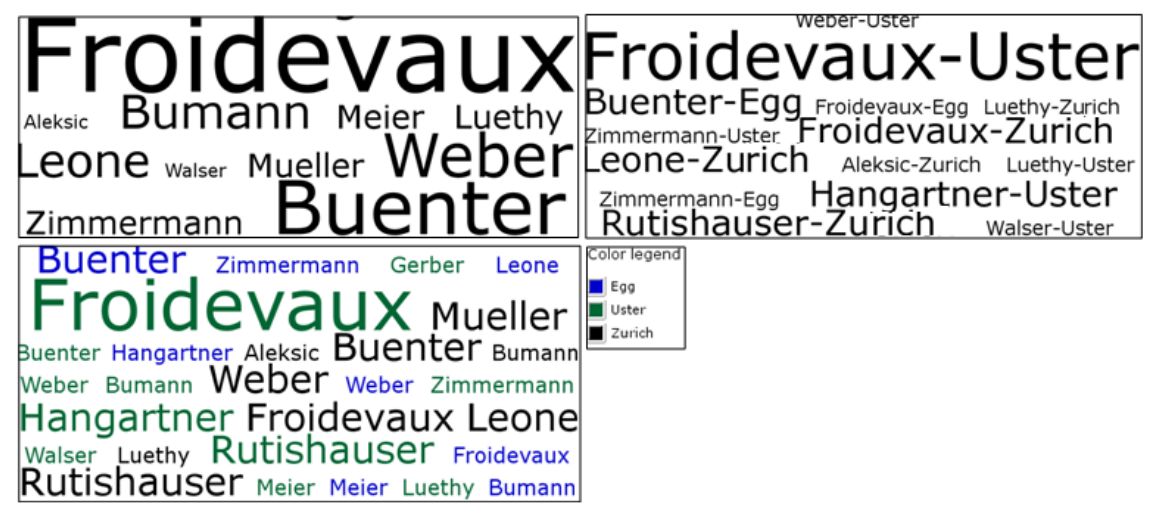

Fig. 4. Exploiting tag cloud features

As already mentioned, we offer different modes for attribute value visualisation and this is depicted in Figure 4. In the data cloud in the upper-left corner, the Contacts collection is visualised by last names. In the data cloud in the upper-right corner, the tag content consists of a combination of two attributes, namely the attribute lastname of Contacts as well as the attribute city of the associated location objects. The tags thus represent the number of contacts with a given name that live in the same city. In this example data set, the tag Froidvaux-Zurich represents the set of contacts with last name Froidvaux who live in Zurich. As one can see in this example, more people with the name Froidvaux live in Uster than in Zurich. For the data cloud in the lower-left corner of Figure 4, we added colour as an additional visualisation dimension. The attribute lastname is bound to the tag content, while the attribute city from the associated location is bound to the colour feature, as already explained before. Note that care has to be taken in choosing the right attributes to bind to the colour feature since, as mentioned previously, it only makes sense if the distinct set of values is small in order that the colour index is of reasonable size and the tag colours are informative. 


\section{Architecture and Implementation}

Since a key objective of this work was to experiment with different tag cloud visualisations and investigate the extent to which these could be used to replace traditional database query languages, it was important that we developed a framework that could serve as an experimental platform. Further, we wanted to be able to experiment with not only different forms of interfaces, but also different database technologies. We therefore implemented a general framework for visualising data from various data sources. The framework is implemented in Java with a JavaFX2 graphical user interface (GUI). Figure 5 gives an overview of the system architecture. The manager component is the heart of the system and responsible for handling requests from the GUI, forwarding these to the database through the database adapter and invoking the visualisation manager to transform the results into the appropriate visual elements to be returned and displayed in the GUI.

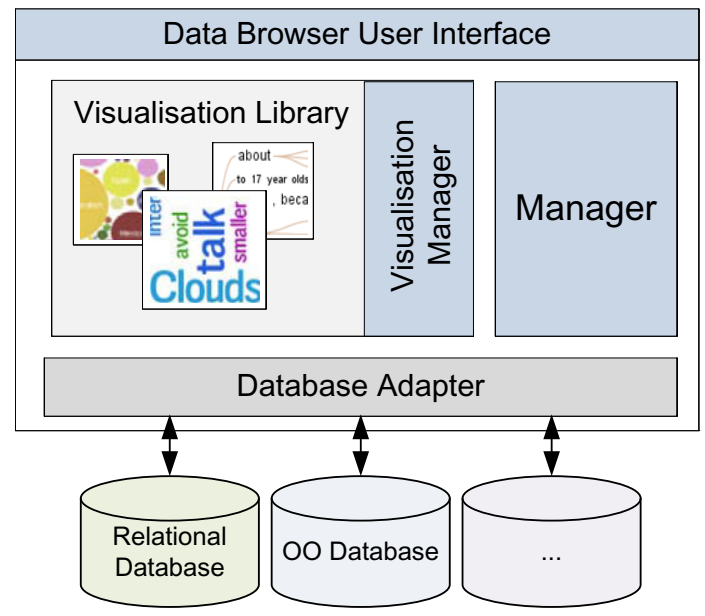

Fig. 5. System Architecture

Our framework is extensible in multiple ways. Firstly, we provide a data adapter interface which can be implemented for any data source. At the moment, we have an implementation for the object databases db4object 3 , OMS Avon 4 and OMSPro5 as well as a MySQL implementation. Secondly, the visualisation manager can manage different kinds of visualisation techniques. Therefore, we provide a visualisation interface which has to be implemented to add a new technique to the visualisation library. We currently provide two tag cloud versions, an earlier version where metadata is selected from a drop down menu and

\footnotetext{
2 http://javafx.com/

3 http://www.db4o.com/

4 http://maven.globis.ethz.ch/projects/avon/

5 http://www.globis.ethz.ch/research/oms/platforms/omspro
} 
a later version that offers synchronised browsing of data and metadata. We have implemented the tag cloud generation algorithm based on [12]. In addition, we are currently working on a bubble chart visualisation.

Our data browser application is flexible and configurable and is currently used as a platform for experimentation in our research group. We have for example integrated it into an ambient information system where researchers were profiled using tag clouds [3].

\section{User Study}

We undertook a preliminary user study to evaluate our system and test the hypothesis that tag clouds are an easy, suitable and intuitive way for users to browse structured data. We recruited ten participants that regularly browse the Web, from which five were computer science professionals and the other five were regular end users. The five computer science professionals included three undergraduate students, a PhD student and a software engineer. The non-expert users' professions were very diverse, including a carpenter, a shop assistant and a human resource and supply chain manager. The ages ranged from 23 to 58 with three participants in their 50 s.

The user study was carried out in individual sessions with each participant. In the first part of each session, we asked the participants for their personal details such as name, age and profession. Additionally, we recorded their computer skills. We were interested in their level of expertise in relational and object database techniques and whether they were familiar with the concept of tag clouds.

In the second part of the session, a participant received a short oral introduction to our data browser. We briefly described the concept of tag clouds to the ones that were not familiar with it and showcased the use of the browser by means of a simple example where the colour feature was used. These oral explanations were intentionally kept very short, since one of our goals was to show that our browser is easy and intuitive to use. The participants were then asked to solve five tasks autonomously by using the data browser. Each of these tasks was targeted at a different degree of difficulty and different concepts of our data browser were needed to answer the question. There was no restriction in the time available to the participants to complete the task.

The participants were asked to browse the contacts database presented in Sect. 3 and find results to the following queries:

- How many persons from ETH have "Bunter" as their last name?

- How many of these persons live in Zurich?

- Get the email addresses of the contacts named "Leone"?

- Where are these contacts situated?

- What is the most frequent age of all contacts?

Finally, the participants had to fill out a questionnaire where we asked about their impressions and experiences with the browser. Questions included whether the short introduction was sufficient help to solve the tasks, whether the tasks 
were difficult to solve, and whether they generally liked the browser. For these questions, they could tick yes or no and provide free text comments.

For the study, we used an early version of the data browser, where the metadata was represented in a drop down menu, from which the user could simply select a collection of objects to be displayed as a data cloud. This is conceptually similar to the synchronised version, where a user selects which collection to represent as a data cloud from the schema cloud.

The participants' previous knowledge and the user study results are summarised in Table 1 and 2 Note that the questions are grouped based on their answers' scale type. For the evaluation of the results, we have divided the participants into two distinct categories, one for the five computer science professionals and one for the five non-experts. We asked them to rate their skills in both relational (RDBMS) and object database (OODBMS) techniques on a scale from 1 to 5 where 1 refers to no skills and 5 to expert skills. While computer scientists were highly skilled in both RDBMS and OODBMS technologies, with average skill levels of 4 and 3.6 respectively, non-experts did not have any previous knowledge in that area, except for one participant who occasionally had to work with an MS Access database. The most surprising fact was that nearly all participants solved the five tasks successfully as indicated in Table 1 question 3. While non-experts accomplished the given tasks successfully, one expert had difficulties with two of the five tasks.

Table 1. Interval Scale

\begin{tabular}{|l|l|l|l|c|c|}
\hline$\#$ & Question & Category & User Group & Average & Std. Dev. \\
\hline \hline \multirow{2}{*}{2.1} & RDBMS Knowledge & \multirow{2}{*}{ Skills } & non-experts & 1.4 & 0.55 \\
\cline { 4 - 6 } & & & computer scientists & 4 & 0.71 \\
\hline 2.2 & OODBMS Knowledge & \multirow{2}{*}{$3 k i l l s$} & non-experts & 1 & 0 \\
\cline { 4 - 6 } & & & computer scientists & 3.6 & 1.14 \\
\hline 3 & \multirow{2}{*}{ Queries } & \multirow{2}{*}{ Task } & non-experts & 5 & 0 \\
\cline { 4 - 6 } & & computer scientists & 4.6 & 0.89 \\
\hline
\end{tabular}

Surprisingly, the familiarity of tag clouds was not as high as we had assumed before the study (Table 2, Question 2.3). While the concept was unknown to all the non-experts, four out of five computer scientists were familiar with tag clouds. This is shown by the significance ( $p$-value) of the CHI-square test that indicates a statistically significant relationship between group membership and tag cloud familiarity. For all the other questions, the CHI-square test showed the a user's expertise had no influence on the answers. Regardless of their computer skills and prior tag cloud knowledge, both experts and non-experts found the provided introduction sufficient for solving the given tasks and none of them found the tasks too difficult. The colour feature was used by five participants, that is three non-experts and two expert users. Nine out of ten participants liked to browse the database with our data browser. One participant thought that the data browser has great potential and another participant commented that he 
Table 2. Nominal Scale

\begin{tabular}{|c|c|c|c|c|c|c|}
\hline \# & Question & Category & User Group & $\overline{\text { Yes }}$ & $\mathrm{No}$ & CHI-Square (p-value) \\
\hline \multirow[t]{2}{*}{2.3} & \multirow[t]{2}{*}{ Tag Cloud Concept } & \multirow[t]{2}{*}{ Skills } & non-experts & 0 & 5 & \multirow[t]{2}{*}{0.048} \\
\hline & & & computer scientists & 4 & 1 & \\
\hline \multirow[t]{2}{*}{4.1} & \multirow[t]{2}{*}{ Sufficient Help } & \multirow[t]{2}{*}{ Feedback } & non-experts & 5 & 0 & \multirow[t]{2}{*}{1} \\
\hline & & & \begin{tabular}{|c|} 
computer scientists \\
\end{tabular} & 4 & 1 & \\
\hline \multirow[t]{2}{*}{4.2} & \multirow[t]{2}{*}{ Task difficulties } & \multirow[t]{2}{*}{ Feedback } & non-experts & 0 & 5 & \multirow[t]{2}{*}{1} \\
\hline & & & computer scientists & 0 & 5 & \\
\hline \multirow[t]{2}{*}{4.3} & \multirow[t]{2}{*}{ Colour Feature } & \multirow[t]{2}{*}{ Feedback } & non-experts & 3 & 2 & \multirow[t]{2}{*}{1} \\
\hline & & & \begin{tabular}{|c|} 
computer scientists \\
\end{tabular} & 2 & 3 & \\
\hline \multirow[t]{2}{*}{4.4} & \multirow[t]{2}{*}{ Liked browser } & \multirow[t]{2}{*}{ Feedback } & non-experts & 4 & 1 & \multirow[t]{2}{*}{1} \\
\hline & & & computer scientists & 5 & 0 & \\
\hline
\end{tabular}

finds the query refinement process very useful. A third participant mentioned that she liked the browser, mainly because one can filter the data by selections, instead of having to write queries.

\section{Discussion}

Our preliminary user study has shown promising results regarding our hypothesis that the use of tag clouds for the browsing and querying of structured data is easy and intuitive. All non-expert users were able to complete all of the given tasks successfully, even though they were not familiar with the concept of tag clouds before participating in the study. Interestingly, the only user who failed to complete the tasks was a computer science professional who failed to correctly complete two out of the five tasks. These results show that our data browser approach has the potential for providing non-experts with an easy and userfriendly means of accessing traditional databases without prior knowledge of a query language and also the actual database schema. Indeed, these are initial findings only and an extended user study has to be carried out that measures efficiency and effectiveness of our approach compared to conventional tools before our hypothesis can be fully validated. However, we believe that there are a number of open issues that merit further investigation. One of these is the question of how best to exploit tag features other than size, such as the tag colour, to help the user to browse the data. Since only $50 \%$ of the participants from both user groups made use of the colour feature to solve the tasks, there is no significant dependency between the use of this feature, the user groups and the accomplishment of the given tasks. This feature would have to be evaluated in a separate study, where more emphasis would be put on exploiting the tag colour for query answering. In addition, we already noted that care has to be taken when using the colour feature, since its use is only helpful if the set of distinct attribute values for a given attribute is rather small and this is something of which users would have to be aware. One participant mentioned that the colour feature is not intuitive and he had problems understanding it in the beginning. 
Other issues to investigate include that of using schema clouds alongside data clouds and whether users would find this more intuitive than selection from menus. In fact, it remains to be seen just how much could be expressed using only tag clouds without the need for menus and check lists and whether this would be beneficial or detrimental to the ease of use.

An interesting hypothesis for further investigation is the question of whether the use of tag clouds would enhance developers' efficiency and effectiveness. While we have shown that non-experts and experts are equally capable of solving a set of given tasks using our browser, we only relied on the use of tag clouds without involving any querying. In an additional study targeted at expert users, one could experiment with a combination of a traditional query interface and tag clouds, where a developer could familiarise themself with the schema and data by browsing it using the tag cloud and iteratively constructing queries. One could then test and compare the quality and time used to construct a query using only a query language with the combined approach. In fact, one of the expert users commented that he likes the option for querying and that the study would have been more meaningful to him if they could first solve the tasks with a traditional database interface and then compare it to the data browser.

While the study reported here focused on browsing structured data sets, we are now experimenting with the browsing of both structured and semi-structured data in terms of a publications database. Based on this, we would like to compare our approach with previous approaches such as [9] where synchronised tag clouds were used to explore semi-structured clinical trial data.

Finally, we note that while our work has focussed mainly on object databases to date, concepts similar to those proposed for data clouds in 6 6 could be adopted to return entities rather than attribute values for specific applications relying on relational databases.

\section{Conclusion}

We have presented a data-driven approach to the browsing and querying of databases based on the visualisation of both data and metadata as tag clouds. We also reported on a preliminary user study involving both expert and nonexpert users which demonstrated the feasibility of the approach. While this initial study provided very positive feedback, we are well aware that there are many open issues and consider the results to be a positive indicator of the potential of tag clouds as a visualisation scheme rather than as validation of a paticular interface design. We therefore plan to further experiment with different tag cloud visualisations as well as how these can be combined into a database browsing interface for both semi-structured and structured data.

\section{References}

1. Bateman, S., Gutwin, C., Nacenta, M.: Seeing Things in the Clouds: The Effect of Visual Features on Tag Cloud Selections. In: Proc. ACM Conf. on Hypertext and Hypermedia (HT 2008), pp. 193-202 (2008) 
2. Farrell, S., Lau, T., Nusser, S., Wilcox, E., Muller, M.: Socially augmenting employee profiles with people-tagging. In: Proc. ACM Symposium on User Interface Software and Technology (UIST 2007), pp. 91-100 (2007)

3. de Spindler, A., Leone, S., Geel, M., Norrie, M.C.: Using tag clouds to promote community awareness in research environments. In: Luo, Y. (ed.) CDVE 2010. LNCS, vol. 6240, pp. 3-10. Springer, Heidelberg (2010)

4. Jagadish, H.V., Chapman, A., Elkiss, A., Jayapandian, M., Li, Y., Nandi, A., Yu, C.: Making Database Systems Usable. In: Proc. ACM SIGMOD Intl. Conf. on Management of Data (SIGMOD 2007), pp. 13-24 (2007)

5. Catarci, T., Costabile, M.F., Levialdi, S., Batini, C.: Visual query systems for databases: A survey. Journal of Visual Languages \& Computing 8(2), 215-260 (1997)

6. Koutrika, G., Zadeh, Z.M., Garcia-Molina, H.: Data Clouds: Summarizing Keyword Search Results over Structured Data. In: Proc. 12th Intl. Conf. on Extending Database Technology (EDBT 2009), pp. 391-402 (2009)

7. Koutrika, G., Zadeh, Z.M., Garcia-Molina, H.: CourseCloud: Summarizing and Refining Keyword Searches over Structured Data. In: Proc. 12th Intl. Conf. on Extending Database Technology (EDBT 2009), pp. 1132-1135 (2009)

8. Kuo, B.Y.L., Hentrich, T., Good, B.M., Wilkinson, M.D.: Tag Clouds for Summarizing Web Search Results. In: Proc. Intl. Conf. on World Wide Web (WWW 2007), pp. 1203-1204 (2007)

9. Hernandez, M.E., Falconer, S.M., Storey, M.A., Carini, S., Sim, I.: Synchronized tag clouds for exploring semi-structured clinical trial data. In: Proc. Conf. of the Center for Advanced Studies on Collaborative Research (CASCON 2008), pp. 42$56(2008)$

10. Rivadeneira, A.W., Gruen, D.M., Muller, M.J., Millen, D.R.: Getting our Head in the Clouds: Toward Evaluation Studies of Tag Clouds. In: Proc. Intl. Conf. on Human Factors in Computing Systems (CHI 2007), pp. 995-998 (2007)

11. Schrammel, J., Leitner, M., Tscheligi, M.: Semantically Structured Tag Clouds: An Empirical Evaluation of Clustered Presentation Approaches. In: Proc. Intl. Conf. on Human Factors in Computing Systems (CHI 2009), pp. 2037-2040 (2009)

12. Kaser, O., Lemire, D.: Tag-Cloud Drawing: Algorithms for Cloud Visualization. In: Tagging and Metadata for Social Information Organization Workshop, in Conjunction with WWW 2007 (2007) 